\title{
OVEREXPRESSION OF MATRIX METALLOPROTEINASE-12 (MMP-12) AS A DIAGNOSTIC BIOMARKER FOR HEPATOCELLULAR CARCINOMA
}

\section{Lobna Mohammed ${ }^{1}$, Mohammed Y. Abdel-kareem ${ }^{2}$, Moustafa A. Sakr ${ }^{1 *}$, Essam Elshemi ${ }^{2}$}

\author{
1Department of Molecular Diagnostics and Therapeutics, Genetic Engineering and Biotechnology \\ Research Institute (GEBRI), University of Sadat city, Menoufia, Egypt \\ ${ }^{2}$ Hepatology department-National Liver Institute - Menoufia University, Egypt \\ *Corresponding author: Email: mostafa.sakr@gebri.usc.edu.eg.
}

\begin{abstract}
Matrix metalloproteinase-12(MMP-12) is involved in tumor invasiveness and metastasis and significantly over-expressed in tissues of HCC. The Aim was to investigate the diagnostic and prognostic value of non-invasive MMP-12 expression in human HCC patients from whole blood.The study was conducted on; 50 patients with chronic HCV related HCC, 50 patients with HCV related cirrhosis and 50 healthy persons as a control group recruited from the National Liver institute, Menoufia University, Egypt. All patients were subjected to tri-phasic CT abdomen when indicated, liver profile, AFP and Molecular characterization of metalloproteinase-12 expression by (qRT-PCR) quantitative real time PCR. We found that, there was statistically significant differences between HCC and cirrhotic patients versus control group regarding CBC parameters and liver profile, ( $p$ - value < 0.01 ), while there was no differences between all groups regarding creatinin ( $p$ - value $>0.05)$. There was a statistically significant difference between HCC patients and other groups regarding mRNAMMP12 ( $p$ - value < 0.01 ). The Sensitivity of MMP12 to detect HCC cases was $72.0 \%$, and specificity $60.0 \%$, with accuracy $70.9 \%$, P value $<0.01$, mRNA-MMP12 and/or AFP had sensitivity of $84.0 \%$, specificity $60.0 \%$, PPV of $51.2 \%$, NPP $88.2 \%$ and Accuracy $71.3 \%$. In Conclusion: mRNA-MMP12 is a good sensitive, bad specific but accurate in diagnosing HCC. Adding mRNA-MMP12 to AFP improves early diagnosis and hence better prognosis.
\end{abstract}

Keywords: HCV, HCC, MMP12, Egyptian patients

\section{INTRODUCTION}

Hepatocellular carcinoma ( $\mathrm{HCC})$ is the commonest primary cancer of the liver. It had risen to become the 5th commonest malignancy worldwide; and the third leading cause of cancer-related deaths. Due to the current epidemic of hepatitis $C$ virus and nonalcoholic fatty liver disease (Gomaa et al.,
2013); HCC incidence is increasing leading to approximately 690,000 new deaths per year (Ferlay et al., 2008).

In Egypt $\mathrm{HCC}$ is one of the three frequently diagnosed cancers (Abdel-Wahab et al., 2000). In patients with HCC, unlike most solid tumors, the coexistence of two life- 
threatening conditions (cancer and cirrhosis) complicates prognostic assessments and the prognosis of patients is solely related to tumor stage and liver condition that simultaneously determine the applicability and efficacy of therapy. Accordingly, early diagnostic modeling and a prognostication art are strongly needed depending on four tightly related aspects: tumor stage, synthetic power of liver, general patient's condition, and the efficacy of therapeutic modality (Llovet et al., 2008).

Alpha fetoprotein (AFP) and real time abdominal ultrasound have been used for long time in the surveillance and diagnosis of HCC. Additionally AFP can be used in follow up after loco-regional and systemic therapy; however it is insufficiently sensitive or specific (Mucozu et al., 2008). It is elevated in many settings of various malignancies in addition to $\mathrm{HCC}$, including germ cell tumors, gastric cancer, and intrahepatic cholangiocarcinoma. Its level also increased in non malignant lesions as in cirrhotic patients without HCC; as a reflection of the degree of necro-inflammatory changes (Kuper et al., 2001; Ranjit 2016; Gonzalez et al., 2015). So looking for other tumor markers is mandatory for patients and hepatologists aiming for earlier diagnosis at earlier stages of the disease. The matrix metalloproteinases (MMP) are family of zinc -dependent endopeptidase enzymes. Its activity was first detected in mouse peritoneal macrophageconditioned media in 1975 , and a $22-k d$ protein responsible for this activity was isolated in 1981. It degrades elastin and a broad range of matrix and non matrix substrates including type IV collagen, type I gelatin, fibronectin, laminin, entactin, vitronectin, proteoglycan, myelin basic protein, and a1-antitripsin. It also associated with tumor invasion and metastasis (Sica et al., 2010) their association with angiogenesis remains controversial (Casazza et al., 2014).

Matrix metalloproteinases, MMP-12 plays an important role in cancer development and progression, and highly expressed in a wide range of cancers, including colorectal, gastric, nasopharyngeal and lung cancers. MMP-12, (human macrophage metalloelastase (HME), identified and formerly isolated from alveolar macrophages of cigarette smokers (Powell and Matrisian 1996; Shapiro et al., 1993) and directly expressed in HCC (GorrinRivas et al., 1998). The expression of MMP-12 is strongly associated with in situ generation of human angiostatin (potent inhibitor of angiogenesis). That inhibits the growth of tumor metastases by suppression of the neovascularization and increased of apoptosis (Yang et al., 2012).The possible carcinogenic role of MMP-12 might related to the single nucleotide polymorphisms of MMP-12-82A $>\mathrm{G}$ (rs2276109) in the promoter region could affect transcriptional activity and lead to alterations in MMP-12 gene expression (Egeblad and werb 2002).

The aim of this study was to investigate the diagnostic and prognostic value of non invasive MMP-12 expression in human HCC patients using whole blood.

\section{SUBJECTS AND METHODS}

SUBJECT

National Liver Institute, Menoufia University, Egypt is a major tertiary center for liver disease; the study was carried; on 50 patients with previously diagnosed HCC aged 47 to 71 years old versus 50 patients with 
chronic HCV related liver cirrhosis aged from 45 to 69 years old without HCC and 50 healthy persons aged from 41 to 65 years old, collected from January 2016 to April 2016. We excluded all patients with cirrhosis or HCC non $\mathrm{HCV}$ related. All participants in the project were approved orally and given oral consent of approval to participate in this study and to give us needed information and blood sample for research.

\section{METHODS}

All of the HCV and HCC patients were have positive of serum hepatitis $C$ virus identified by serology and confirmed by qualitative PCR to detect HCV-RNA load. The level of Alfa fetoprotein (AFP) was tested for all samples to detect the early stages of HCC. Abdomen Triphasic CT scan was done for patients with $\mathrm{HCC}$ to determine the stage of the HCC. Biochemical and hematological parameters were performed for all samples.

\section{QUANTITATIVE REAL - TIME - PCR (QRT- PCR) FOR MMP12}

Blood samples of the HCC, HCV and control groups were obtained by vein-puncture on EDTA tubes as anticoagulant. The collected whole blood immediately frozen at $-80^{\circ} \mathrm{C}$ until analyzed.Total RNA was isolated from whole blood by PureLink RNA mini kit (Thermos scientific. USA) according to the manufacturer's instructions. The first strand cDNA was synthesized with HiSenScriptRH(-) cDNA synthesis kit (iNTRON, Korea) using 1 $\mu \mathrm{g}$ RNA. The Real-time PCR was done using RealMOD Green Real-time PCR Master Mix Kit (iNTRON, Korea). QRT-PCR to examine relative MMP12 mRNA level was performed using GAPDH as an internal control and the
MMP12 mRNA was expressed as fold change to gapdh.

\section{STATISTICAL ANALYSIS}

Data was statistically analyzed using SPSS (statistical package for social science) program version $2, \mathrm{p}$ value $<0.05$ was considered statistically significant: Data are shown as mean, range or value and 95\% confidence interval $(95 \% \mathrm{Cl})$ and frequency and percent. All data were tested with Kolmogorov- Smirnov $\mathbf{Z}$ test and most of them were found normally distributed and so presented with mean \pm SD and was used parametric testes on doing association or correlation. ANOVA test was done to compare three variables; one qualitative variable and the other two are quantitative variables of normally distributed variable to detect mean and standard deviation where LSD test is a post hoc test was done to detect the relationship between variables within groups. Kruskal-Wallis test was done to compare three or more variables; one qualitative variable and the other variables are quantitative variables of none normally distributed variables to detect mean and standard deviation where Tamhane test post hoc test were done to detect the relationship between variables within groups. Roc curve (Receiver operating characteristic curve): was done to detect cut off level of any tested variable where at this level there is a the best sensitivity and specificity cut off values of the variables for the presence of the disease moreover,. The validity of the model was measured by means of the concordance (c) statistic (equivalent to the area under the Roc curve). A model with a cut off value above 0.7 is considered useful while a cut off value 
between 0.8 and 0.9 indicated excellent diagnostic accuracy.

\section{RESULTS AND DISCUSSION}

This study was conducted on 3 groups of patients 50 patients with $\mathrm{HCC}, 50$ patients with liver cirrhosis without HCC (LC) and 50 healthy persons (normal) group with matched age and sex.

Demographic data, biochemical, and hematological characteristics of the studied groups

The values of the hematological, and biochemical variables of the groups under study are summarized in Table $(\mathbf{1 , 2 )}$.. Serum levels of ALT, AST, SGOT, total bilirubin, and INR were significantly higher in HCC than other groups; whereas albumin, hemoglobin levels, WBCs count and platelets count were significantly lower in HCC patients versus the other groups. Creatinine was not significantly different between studied groups.

Table 1: the differences between studied groups regarding hematological parameters

\begin{tabular}{|c|c|c|c|c|c|}
\hline Variables & $\begin{array}{c}\text { HCC } \\
(\mathrm{N}=14) \\
\text { Mean } \pm S D\end{array}$ & $\begin{array}{c}\text { HCV } \\
(\mathrm{N}=12) \\
\text { Mean } \pm S D\end{array}$ & $\begin{array}{c}\text { Control } \\
(\mathrm{N}=10) \\
\text { Mean } \pm \text { SD }\end{array}$ & ANOVA & $\mathbf{P}$ \\
\hline $\mathbf{H b}$ & $11.7350 \pm 1.79851$ & $13.57 \pm 0.55539$ & $12.7300 \pm 0.71611$ & 20.399 & $0.000^{*}$ \\
\hline RBCs & $3.8750 \pm 0.68491$ & $4.4650 \pm 0.25374$ & $4.3625 \pm 0.22610$ & 26.275 & 0.674 \\
\hline WBCs & $5.2925 \pm 2.25120$ & $6.7050 \pm 0.62345$ & $6.0625 \pm 0.91419$ & 20.140 & 0.001 \\
\hline PLT & $\begin{array}{c}129.4750 \\
\pm 62.36287\end{array}$ & $\begin{array}{c}237.00 \pm \\
29.93155\end{array}$ & $\begin{array}{c}335.0000 \\
\pm 54.30210\end{array}$ & 42.984 & $0.000^{* *}$ \\
\hline Time & $\begin{array}{c}13.5125 \pm \\
0.88468\end{array}$ & $\begin{array}{c}12.7250 \pm \\
0.71567\end{array}$ & $\begin{array}{c}12.0000 \pm \\
0.00000\end{array}$ & 53.033 & $0.000^{*}$ \\
\hline Con & $78.2250 \pm 11.60125$ & $89.1500 \pm 9.90351$ & $100.0000 \pm 0.00000$ & 61.137 & $0.000^{*}$ \\
\hline INR & $1.2500 \pm 0.17394$ & $1.1300 \pm 0.11810$ & $1.0000 \pm 0.00000$ & 61.137 & $0.000^{*}$ \\
\hline
\end{tabular}

Table 2: The difference between studied groups regarding the biochemical parameters

\begin{tabular}{|c|c|c|c|c|c|}
\hline & $\begin{array}{c}\text { HCC } \\
(\mathrm{N}=50) \\
\text { Mean } \pm S D\end{array}$ & $\begin{array}{c}\text { HCV } \\
(\mathrm{N}=50) \\
\text { Mean } \pm S D\end{array}$ & $\begin{array}{c}\text { Control } \\
(\mathrm{N}=50) \\
\text { Mean } \pm S D\end{array}$ & ANOVA & $\mathbf{P}$ \\
\hline Creatinine & 0.90750 .40471 & $0.7500 \quad 0.09871$ & $0.6500 \quad 0.11323$ & $2.428^{*}$ & 0.119 \\
\hline SGPT & $\begin{array}{c}51.8250 \\
21.73133\end{array}$ & $\begin{array}{l}35.4500 \\
7.58862 \\
\end{array}$ & $\begin{array}{r}20.3750 \\
5.54556\end{array}$ & $13.42^{*}$ & $0.000^{* *}$ \\
\hline SGOT & $\begin{array}{r}52.6500 \\
24.51640\end{array}$ & $\begin{array}{c}38.000 \\
10.10204\end{array}$ & $\begin{array}{l}19.3750 \\
4.32457\end{array}$ & $4.676^{*}$ & $0.031^{*}$ \\
\hline Bilirubin & $\begin{array}{lll}0.9637 & 0.32005\end{array}$ & $0.6800 \quad 0.06869$ & $0.6000 \quad 0.07161$ & 39.050 & $0.000^{\star *}$ \\
\hline Albumin & 3.34750 .50127 & $3.7550 \quad 0.13195$ & $3.8750 \quad 0.13156$ & 32.079 & $0.000^{\star *}$ \\
\hline
\end{tabular}




\section{AFP AND HGF EXPRESSION LEVELS IN THE STUDIED GROUP}

As shown in Table 3 and Figure 1 the level of the MMP12 detected by qRT-PCR in HCC cases and other groups normalized GAPDH (housekeeping gene) as internal control. We found that the expression level of MMP12 was significantly higher in chronic hepatitis $\mathrm{C}$ and HCC in comparison with healthy control group $(p=0.000)$. Whereas the median level of serum AFP showed a significant higher value in HCC group versus $\mathrm{HCV}$, and healthy control groups ( $P$ $=0.000$ ).our findings demonstrate that, MMP12may be used as a potential HCC biomarkers.

Table 3.Comparison of the median values of fold change of the studied MMP12 and AFP median values as markers for HCC

\begin{tabular}{|l|c|c|c|c|c|}
\hline & $\begin{array}{c}\text { HCC } \\
(\mathbf{N}=50) \\
\text { Mean } \pm \text { SD }\end{array}$ & $\begin{array}{c}\text { HCV } \\
\mathbf{( N = 5 0 )} \\
\text { Mean } \pm \text { SD }\end{array}$ & $\begin{array}{c}\text { Control } \\
(\mathbf{N}=50) \\
\text { Mean } \pm \text { SD }\end{array}$ & Kw & P-value \\
\hline AFP & $229.2595 \pm 419.99$ & $3.3275 \pm 1.32645$ & $1.0000 \pm 0.358$ & 41.173 & $0.000^{*}$ \\
\hline Log MMP12 to GAPDH & $3.2292 \pm 5.76778$ & $2.4877 \pm 3.78317$ & $0.9862 \pm 1.548$ & 28.136 & $0.000^{*}$ \\
\hline
\end{tabular}

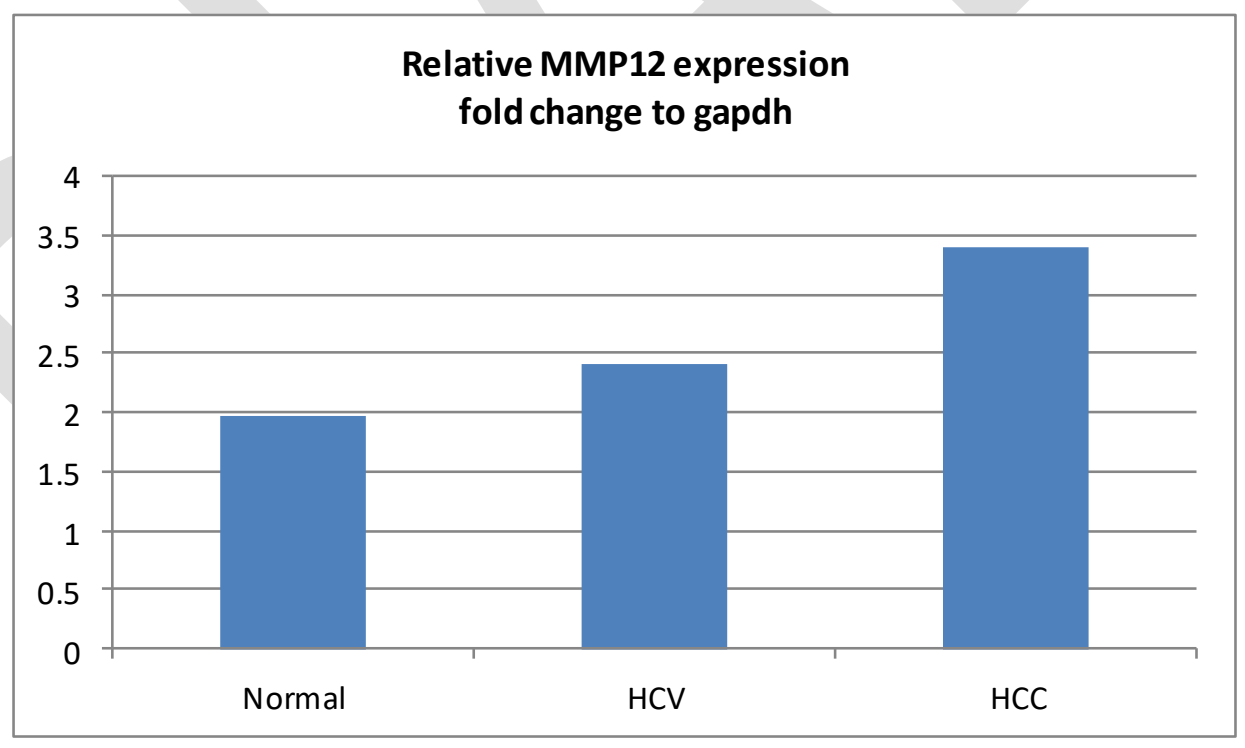

Fig 1. HGF level between the studied groups

MMP12 AS A POTENTIAL TUMOR MARKER FOR HCC

We used ROC curve analysis to compare the accuracy of noninvasive level of MMP12 and AFP to predict patients with HCC. It was found that MMP12 could predict HCC at level of 2.78 fold with sensitivity $72 \%$ and specificity $60 \%$ with accuracy $70.9 \%$ while with
AFP could predict HCC at level of $90 \mathrm{ng} / \mathrm{dl}$ with sensitivity $75 \%$ and specificity $70 \%$ (Table 4 , Fig 2). When adding MMP12 mRNA expression with AFP level, the sensitivity to detect HCC cases increased to $84 \%$ and accuracy $71.3 \%$. From these results it was clarified that the MMP12 is more sensitive and more accurate when adding to Convential 
tumor marker AFP in prediction of HCC and in turn it will have a better implications on the HCC treatment outcome. Overall, these results suggest that MMP12 could serve as a useful tumor biomarker for prediction of HCC in comparing with, $\mathrm{HCV}$, and healthy control results.

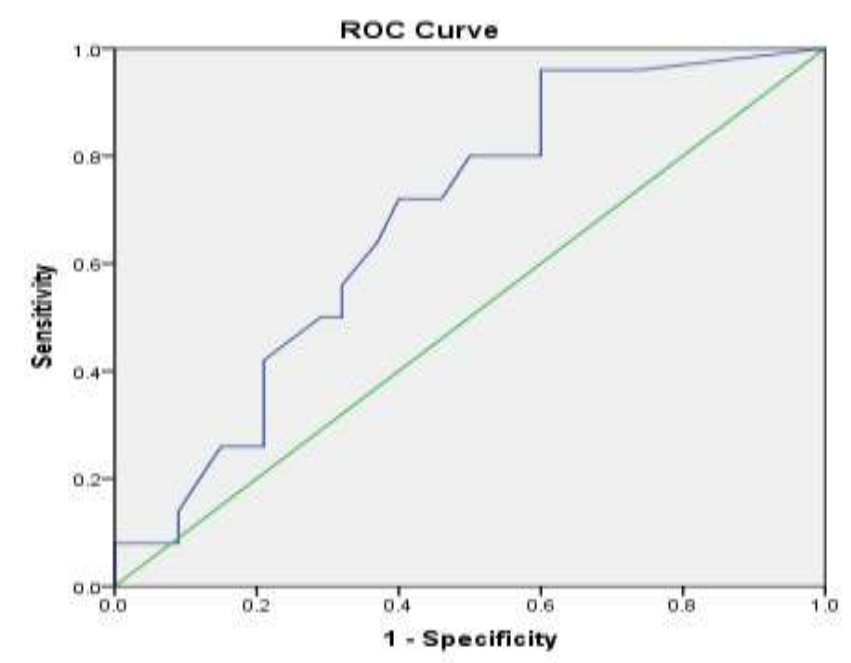

Fig 2. ROC curve of MMP12 for HCC cutoff value

Table 4: Sensitivity, specificity and accuracy of MMP12 expression

\begin{tabular}{|c|c|c|c|c|c|}
\hline Studied variables & Sensitivity & Specificity & AUC & Accuracy & P-value \\
\hline $\begin{array}{c}\text { RT.PCR_for_MMP12 } \\
\text { Cut level } \mathbf{0 . 7 0 8 8}\end{array}$ & $72.0 \%$ & $60.0 \%$ & 0.68 & $70.9 \%$ & $<0.01^{* *}$ \\
\hline MMP12 and AFP & $84.0 \%$. & $60.0 \%$. &.,$v \uparrow$ & $71.3 \%$. & $<0.00^{* *}$ \\
\hline
\end{tabular}

Prognosis for HCC depends mainly on early diagnosis that generally related to tumor stage at presentation that guides treatment decisions. The 4 key factors that may affect the prognosis include: (Gomaa et al., 2013) tumor stage at diagnosis; (Ferlay et al., 2008) overall health of the patient; (Abdel-Wahab et al., 2000) hepatic synthetic function; and (Llovet et al., 2008) efficacy of treatment (Bruix et al., 2000). So patients and clinician are in need for an ideal tumor marker that can accurately detect HCC in high risk patients as cirrhotic patients and patients with chronic HBV infection at early stages. Tumor biomarkers are indicators of a physiological status, detectable in serum, urine, or tissues for patients with cancer. Ideal biomarkers; its presence and changes in body fluids can reflect the disease occurrence, progression, recurrence and prognosis. Serum Alpha fetoprotein (AFP) is produced by embryonic liver cells during pregnancy and decreases after birth. It is one of the most famous serum biomarkers; however, the specificity and reliability of AFP in diagnosis of $\mathrm{HCC}$ is questioned (Durazo et al., 2008, Marrero et al., 2009).

Our study discussed the utility of MMP-12 in synergism with AFP for diagnosis of HCC. We prospectively evaluated 50 patients with HCC related cirrhosis post 
chronic HCV infection, diagnosed in our outpatients or inpatients clinics in NLI using the standard diagnostic tools as viral markers, liver and kidney profiles, AFP and all patients have the criteria of HCC in tri-phasic CT abdomen studies, we compared the serum of those patients to another 2 groups, the first one was patients with chronic HCV infection related cirrhosis but without HCC (proved by low level serum AFP and no detectable focal lesions by abdominal ultrasound). If there's any doubt about the diagnosis tri-phasic CT abdomen was done to exclude HCC, the second group was healthy persons. We evaluated the serum level of MMP-12 in all studied groups.

We found in our study that, normal persons had significantly better parameters than the cirrhotic patients and HCC in all CBC component, liver transaminases, PT, concentration and INR in addition to total proteins and albumin ( $p$ - value $<0.01$ ), Table $(1,2)$. We evaluated mRNA expression of MMP-12 in our studied groups and found that the MMP-12 is a good sensitive, bad specific but accurate in diagnosing HCC, Table $(3,4)$. To our knowledge our study was the first one that discussed the diagnostic value of adding over expressed MMP-12 mRNA and AFP in diagnosis of HCC. It was found that MMP12 could predict HCC at level of 2.78 fold with sensitivity $72 \%$ and specificity $60 \%$ with accuracy $70.9 \%$ while with AFP could predict HCC at level of $90 \mathrm{ng} / \mathrm{dl}$ with sensitivity $75 \%$ and specificity $70 \%$ (Table 4, Fig 2). When adding MMP12 mRNA expression with AFP level, the sensitivity to detect $\mathrm{HCC}$ cases increased to $84 \%$ and accuracy $71.3 \%$. From these results it was clarified that the MMP12 is more sensitive and more accurate when adding to Convential tumor marker AFP in prediction of HCC and in turn it will have a better implications on the $\mathrm{HCC}$ treatment outcome.

Many studies discussed the use of MMPs in HCC, GIT cancers and other cancers. Liu et al. hypothesized that the surrounding tumor-associated stromal cells play a crucial role in 14-3-3б-regulated HCC cell invasion. They incubated H68 fibroblasts, THP-1 and phorbol-12-myristate-13-acetate (PMA)-treated THP-1 (PMA-THP-1) cells with conditioned media of control (control-CM) and 14-3-30-

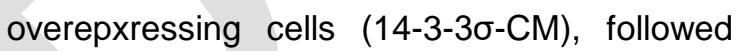
by co-culture with $\mathrm{HCC}$ cells. HCC cells significantly enhanced their invasive ability compared with control-CM treated cells. Moreover, incubation with 14-3-3б-CM induced differential expression profiles of (MMPs) in fibroblasts (MMP-1, MMP-2, MMP-9, MMP-12 and MMP-14), THP-1 (MMP-1 and MMP-12) and PMA-THP-1 cells (MMP-2, MMP-12 and MMP-14) (Liu et al., 2016).

$\mathrm{Ng}$ et al. studied the significance and prognostic value of MMP-12 expression in human HCC. The MMP-12 mRNA level of 139 pairs of tumor and non-tumor liver tissues of HCC patients after hepatectomy. They found that the PCR. MMP-12 mRNA was significantly elevated in tumor tissues compared to nontumor and normal liver tissues. MMP-12 mRNA was over-expressed in $58 \%$ of HCC patients. Over-expression ofMMP-12 mRNA was significantly correlated with presence of venous infiltration $(p=0.004)$, high serum AFP level $(p=0.012)$, early tumor recurrence $(p=0.018)$ and poor overall survival $(p=0.02)$ of HCC patients. Moreover, MMP-12mRNA was an independent factor in predicting overall survival after hepatectomy ( $\mathrm{Ng}$ et al., 2011). Similarly Manuel et al. studied the correlation 
between human macrophage metalloelastase gene expression and angiostatin production using the messenger RNA (mRNA) expression and angiostatin generation in tumorous and non tumorous tissues obtained from $40 \mathrm{HCC}$ patients after partial hepatectomy. The mRNA was detected in 25 out of $40 \mathrm{HCC}$ tissues and, in all of these cases; the expression in tumorous tissues was stronger than in the nontumorous tissues. They concluded that MMP gene was expressed by HCC cells, and it can be responsible for the angiostatin generation by such tumor cells and may serve as a prognostic marker in HCC patient (Manuel et al., 1998).

Miyoshi et al. examined whether Snail gene expression correlates with cancer invasion and prognosis in HCC. RT-PCR was performed to evaluate Snail, E-cadherin, and MMP mRNA expressions in eight nodule-innodule tumors and 47 ordinary HCC tissues. In the nodule tumors, Snail expression significantly increased with tumor dedifferentiation. In the ordinary HCC tissues, Snail expression was significantly correlated with portal vein invasion, intra-hepatic metastasis, correlation with MT1-MMP expression, poorer prognosis by up-regulating MMP expression (Miyoshi et al., 2005).

From the above shown data; MMP -12 might be a universal target for diagnosis and prognosis of cancer and might be used as a promising screening marker in looking for hidden malignancies. Furthermore MMP-12 may play a crucial role in anti cancer therapy. Fucosyltransferase IV (FUT4) has been implicated in cell adhesion, motility, and tumor progression in human epidermoid carcinoma A431 cells. Determination of the effect of FUT4 on MMP-12 induced by EGF in A431 cells. So treatment with EGF resulted in an alteration of cell morphology and induced an increase in the expression of MMP-12 (Yang et al., 2012).

Additionally inhibition of MMP synthesis in anticancer therapy Several agents have been developed that block the of MMPs, prevent them from interacting with the molecules that direct their activities to the cell surface or inhibit their enzymatic activity or can also be reduced indirectly, by inhibition of the signal-transduction pathways that induce MMP transcription by inhibiting tyrosine kinase receptor signaling (Noonberg et al., 2000; , Hidalgo and Eckhardt 2001; , Moore et al., 2002).

Conclusion, the present study revealed that, the expression level of MMP12 was significantly higher in $\mathrm{HCC}$ group than into cirrhotic or the healthy group. Furthermore the sensitivity of the MMP12with AFP to detect the $\mathrm{HCC}$ cases is higher than the sensitivity of the traditional tumor marker (AFP) alone. Thus the expression level of MMP12 can be used as a potential noninvasive tumor marker for HCC patients. Additional studies with high sample size to validate and confirm the results were needed. 


\section{REFERENCES}

Abdel-Wahab M, El-Enein AA, Abou-Zeid M, et al. Hepatocellular carcinoma in Mansoura-Egypt: Experience of 385 patients at a single center. Hepatogastroenterology 2000; 47:663- 68.

Bruix J, Sherman M, Llovet JM, Beaugrand M, Lencioni R, Burroughs AK, Christensen E, Pagliaro L, Colombo $M$ and Rodés J. Clinical management of hepatocellular carcinoma. Conclusions of the Barcelona 2000 EASL conference. European association for the study of the Liver. J Hepatol 2001; 35(3): 421-430.

Casazza A, Di Conza G, Wenes M, Finisguerra V, Deschoemaeker $S$, Mazzone M. Tumor stroma: a complexity dictated by the hypoxic tumor microenvironment. Oncogene. 2014; 33:1743-1754.

Durazo FA, Blatt LM, Corey WG, et al. Des gamma carboxyprothrombin, afetoprotein and AFP-L3 in patients with chronic hepatitis, cirrhosis and hepatocellular carcinoma. J Gastroenterol Hepatol 2008; 23:1541-1548.

Egeblad M, Werb Z. New functions for the matrix metalloproteinases in cancer progression. Nat Rev Cancer VOLUME 2 | MARCH 2002 | 161174.

Ferlay J, Shin HR, Bray F, Forman D, Mathers C, Parkin DM. Estimates of worldwide burden of cancer in
2008: GLOBOCAN 2008. Int. J. Cancer 127: 2893-2917.

Gomaa AI, Khan SA, Toledano MB, Waked I and Taylor- RobinsonSD. Hepatocellular carcinoma: Epidemiology, risk factors and pathogenesis. World J Gastroenterol 2013; 14(27): $43 \cdot 0$ 4308.

Gonzalez SA, Keeffe EB. Diagnosis of Hepatocellular Carcinoma: Role of Tumor Markers and Liver Biopsy. Clin Liver Dis 2011; 15:297-306.

Gorrin-Rivas MJ, Arii S, Furutani M, et al. Expression of human macrophage metalloelastase gene in hepatocellular carcinoma: correlation with angiostatin generation and its clinical significance. Hepatology 1998; 28:986 -993.

Hidalgo, M. \& Eckhardt, S. G. Development of matrix metalloproteinase inhibitors in cancer therapy. J. Nat Cancer Inst. 93, 178-193 (2001).

Kuper Ye W, Broomè U, Romelsjo A, Mucci LA, Ekbom A, Adami HO, Trichopoulos $D$ and Nyren $O$. The risk of liver and bile duct cancer in patients with chronic viral hepatitis, alcoholism or cirrhosis. Hepatology 2001; 34: 714-718.

Liu C, Chang T, Lin Y, Yu Y, Ko B, Sung L, Liou J: Paracrine regulation of matrix metalloproteinases contributes to cancer cell invasion by hepatocellular carcinoma- 
secreted 14-3-3б Oncotarget, 2016, Vol. 7, No. 24; 36988-36999.

Llovet JM, Di Bisceglie AM, Bruix J, Kramer BS, Lencioni R, Zhu AX, Sherman M, Schwartz M, Lotze M, Talwalkar $\mathrm{J}$ and Gores GJ. Design and endpoints of clinical trials in hepatocellular carcinoma. J Natl Cancer Inst 2008; 100(10): 698711.

Manuel J. Gorrn R, Shgeki A, Masharu F, Tomika $\mathrm{H}$, Masaki $\mathrm{M}$. et al. Expression of Human Macrophage Metalloelastase Gene in Hepatocellular Carcinoma: Correlation With Angiostatin Generation and its Clinical Significance. HEPATOLOGY Vol. 28, No. 4, 1998.

Marrero JA, Feng Z, Wang Y, et al. Alphafetoprotein,

Des-gamma Carboxyprothrombin, and LectinBound Alpha-fetoprotein in Early Hepatocellular Carcinoma AFP, DCP, and AFP-L3 in Hepatocellular carcinoma. Gastroenterology 2009; 137(1):110-118.

Miyoshi A, Kitajima Y, KidoS, Shimonishi T, Matsuyama S, Kitahara $\mathrm{K}$ and Miyazaki K: Snail accelerates cancer invasion by up regulating MMP expression and is associated with poor prognosis of hepatocellular carcinoma metastasis, and poor prognosis. British Journal of Cancer (2005) 92, 252-258.

doi:10.1038/sj.bjc.6602266.

Moore, M. J. et al. A comparison between gemcitabine (GEM) and the matrix metalloproteinase (MMP) inhibitor BAY12-9566 (9566) in patients (PTS) with advanced pancreatic cancer. ASCO Online [online] (cited 05 Feb 2002), <http://www. asco. org/prof/me/html/00abstracts/gic/m _930.htm> (2000).

Mucozu T, hidenari N, Matsui D, Kanekawa T and sumino $Y$. Serum VEGF and AFP in HCV-related liver cirrhosis and HCC. Anticancer research 2013; 33: 1013-1022.

$\mathrm{Ng}$ KT, Qi X, Kong KL, Cheung BY, Lo CM, Poon RT, Fan ST, Man K Overexpression of matrix metalloproteinase-12 (MMP-12) correlates with poor prognosis of hepatocellular carcinoma. Eur $J$ Cancer. 2011 Oct;47(15):2299-305. doi: $\quad$ 10.1016/j.ejca.2011.05.032. Epub 2011 Jun 15.

Noonberg, S. B. \& Benz, C. C. Tyrosine kinase inhibitors targeted to the epidermal growth factor receptor subfamily: role as anticancer agents. Drugs 59, 753-767 (2000).

Powell WC, Matrisian LM. Complex roles of matrix metalloproteinases in tumor progression. Curr Top Microbiol Immunol 1996; 213:1-21.

Ranjit Chauhanand Nivedita Lahiri Tissueand Serum-Associated Biomarkers of Hepatocellular Carcinoma Biomarkers in Cancer 2016:8(S1).

Shapiro SD, Kobayashi DK, Ley TJ. Cloning and characterization of a unique elastolytic metalloproteinase produce by human alveolar macrophages. J Biol Chem 1993; 268:23824 -23829. 
Sica A. Role of tumor-associated macrophages in cancer related inflammation. Exp Oncol. 2010; 32:153-158.

Yang XS, Liu SA, Liu JW, Yan Q. Fucosyltransferase IV enhances expression of MMP-12 stimulated by EGF via the ERK1/2, p38 and NF-KB pathways in A431 cells. Asian Pac J Cancer Prev 2012; 13: 1657-62 